\title{
Potential candidate cells for constructing tissue-engineered lacrimal duct epithelium: a histological and cytological study in rabbits*
}

\author{
Chen XIE, Xiu-yi LI, Hong-guang CUI ${ }^{\dagger *}$ \\ (Department of Ophthalmology, the First Affiliated Hospital, School of Medicine, Zhejiang University, Hangzhou 310003, China) \\ ${ }^{\dagger} \mathrm{E}-\mathrm{mail}$ :dr_chg@163.com
}

Received May 11, 2015; Revision accepted Sept. 30, 2015; Crosschecked Oct. 20, 2015

\begin{abstract}
Objective: Injury and deficiency of the lacrimal duct epithelium (LDE) can lead to a variety of lacrimal diseases. The purpose of this study was to characterize potential candidate cells for constructing a tissue-engineered LDE. Methods: Different areas of the conjunctiva and lacrimal duct tissue were removed from male adult New Zealand white rabbits for histological evaluation. Hematoxylin and eosin staining and immunohistochemical staining of cytokeratin AE1+AE3, cytokeratin 4, Ki-67, and MUC5AC were observed by light microscopy. The surface morphologies of different epithelial tissues and cellular structures were examined using field-emission scanning electron microscopy and transmission electron microscopy. Epithelial cells were isolated from tissues and identified by specific markers. In vitro, proliferative ability and Western blot analyses of the proliferating cell nuclear antigen (PCNA) of different epithelial cells cultured in identical environments were investigated and compared. Results: Histologically, the epithelial specific markers, cytokeratin AE1+AE3 and cytokeratin 4, were expressed in the conjunctiva epithelium and the LDE. Notably, highly proliferative cells stained with Ki-67 were concentrated under the epithelium in a dome structure of the posterior palpebral conjunctiva. Differentiated goblet cells were also found to a lesser extent in this region. Primary palpebral and fornical conjunctival epithelial cells (PFCECs), bulbar conjunctival epithelial cells (BCECs), and lacrimal duct epithelial cells (LDECs) were successfully separated from tissues. In vitro, rabbit PFCECs and LDECs grew faster and expressed more PCNA than BCECs. Conclusions: PFCECs are anatomically similar to LDECs. They also have similar morphological characteristics, immune phenotypes, and proliferation features. PFCECs are therefore potential candidate cells to replace LDECs in tissue engineering to treat lacrimal duct diseases.
\end{abstract}

Key words: Conjunctival epithelial cells, Cytokeratin, Lacrimal duct epithelial cells, Mucin, Proliferating cell nuclear antigen, Tissue engineering doi: 10.1631 jzus.B1500113

Document code: A

CLC number: R777.2; Q813

\section{Introduction}

The human lacrimal duct system, consisting of the lacrimal duct, lacrimal sac, and nasolacrimal duct, is an important component of the lacrimal apparatus. Extra tear fluid is drained by the lacrimal ducts into the inferior meatus of the nose. Any stenosis or obstruction in the lacrimal duct system can induce

\footnotetext{
* Corresponding author

* Project supported by the Natural Science Foundation of Zhejiang Province (No. LY12H12009), China

(10) ORCID: Hong-guang CUI, http://orcid.org/0000-0001-9144-2274

(C) Zhejiang University and Springer-Verlag Berlin Heidelberg 2015
}

symptoms of epiphora, chronic ocular surface inflammation, and pyorrhea, which may increase the risk of endophthalmitis after intraocular surgery (Lopez et al., 1993; Prabhasawat and Tseng, 1998; Kam et al., 2014). Various surgical treatments, including traditional or laser-assisted dacryocystorhinostomy, silastic tube intubation, balloon dacryoplasty, and laser lacryocystoplasty, can be applied to alleviate obstruction in lacrimal duct systems (Goldstein et al., 2006; Avgitidou et al., 2015; Takahashi et al., 2015). However, the effectiveness of these treatments depends on the integrity of lacrimal duct mucosa (Takahashi et al., 2013; Baran et al., 
2014). Moreover, with increased trauma and iatrogenic injury of the lacrimal duct mucosa, ophthalmologists must deal with the challenge of limited treatment options (Mukherjee and Dhobekar, 2013).

Langer and Vacanti (1993) first proposed the concept of tissue engineering, an interdisciplinary field that applies the principles of engineering and life sciences for the development of biological substitutes that restore, maintain, and/or improve the functioning of biological tissues and whole organs. Pellegrini et al. (1997) pioneered the restoration of damaged corneal surfaces with autologous tissue-engineered corneal limbal epithelium. Nakamura and coworkers first constructed rabbit and human oral mucosal epithelium in vitro, and reconstructed the ocular surface of corneal limbus stem cell deficiency (LSCD) in rabbit eyes (Nakamura and Kinoshita, 2003; Nakamura et al., 2003). However, tissue engineering for the lacrimal duct epithelium (LDE) has not been investigated.

Easy extraction from tissue is an important condition for seed cells in tissue engineering. However, LDE cells located in the tube structure are not easily obtained in vivo. The conjunctival epithelium $(\mathrm{CjE})$, consisting of palpebral, fornical, and bulbar $\mathrm{CjE}$, maintains the microenvironment on the ocular surface Ex vivo conjunctival grafts have been cultured on amniotic membrane and transplanted into animals and patients with LSCD (Tanioka et al., 2006; Ang et al., 2010; Ricardo et al., 2013). Moreover, the LDE is continuous with the $\mathrm{CjE}$, and expresses a similar broad spectrum of mucins, to reduce drag and enhance tear outflow (Paulsen et al., 2003; Jäger et al., 2007). Therefore, $\mathrm{CjE}$ cells might be the ideal candidate cells to be used for constructing tissue-engineered LDE. In the present study, we compared the biological differences between palpebral, fornical and bulbar $\mathrm{CjE}$ and the LDE of rabbits, both in situ and in vitro, to identify a suitable location of $\mathrm{CjE}$ candidate cells for reconstructing tissue-engineered LDE.

\section{Materials and methods}

\subsection{Animals and epithelial tissue preparation}

The animals used in this study were male adult New Zealand white rabbits (mean age, 12 months; body weight, 2-3 kg; provided by the Experimental Animal Center of Zhejiang University, Hangzhou,
China). All animal handling procedures were approved by the Zhejiang University Animal Care and Use Committee. Rabbits were euthanized by air embolism via a peripheral ear vein. The animals were designated as pathogen free and received regular ocular health checks. Under sterile conditions, three types of ocular epithelial tissues (the palpebral and fornical conjunctiva, from the eyelid margin to the fornix; the bulbar conjunctiva $2 \mathrm{~mm}$ from the fornix to $2 \mathrm{~mm}$ from the limbus; and the entire lacrimal duct tissue) were separated from both sides of the rabbit eye for experimental use. Each experiment was performed at least in triplicate using samples from three rabbits.

\subsection{Hematoxylin and eosin ( $\mathrm{H} \& \mathrm{E})$ staining and immunohistochemistry}

The tissues obtained from euthanized rabbits were fixed in $4 \%$ formalin overnight. After dehydration, the specimens were embedded in paraffin, cut into 5- $\mu \mathrm{m}$ sections, and stained with $\mathrm{H} \& \mathrm{E}$. The histological morphology of each epithelial specimen was observed using light microscopy. Immunohistochemical staining of specific antigens was performed on sections of the conjunctival and lacrimal duct tissues (Bonilha et al., 2006). Deparaffinized 5- $\mu \mathrm{m}$ sections were boiled in $1 \times$ target retrieval solution (Dako, Capenteria, CA, USA) for $20 \mathrm{~min}$. Endogenous peroxidase activity was eliminated by treating samples with 3\% hydrogen peroxide for $15 \mathrm{~min}$. After blocking in $5 \%(0.05 \mathrm{~g} / \mathrm{ml})$ normal goat serum diluted in $1 \times$ phosphate-buffered saline (PBS; $155 \mathrm{mmol} / \mathrm{L} \mathrm{NaCl}$, $1.06 \mathrm{mmol} / \mathrm{L} \mathrm{KH}_{2} \mathrm{PO}_{4}, 2.97 \mathrm{mmol} / \mathrm{L} \mathrm{Na}_{2} \mathrm{HPO}_{4}, \mathrm{pH}$ 7.4), the sections were incubated with mouse anticytokeratin AE1+AE3 antibody (1:100 (v/v); Abcam, Cambridge, MA, USA), mouse anti-cytokeratin 4 (CK4) antibody (1:100 (v/v); GeneTex, Irvine, CA, USA), mouse anti-Ki-67 antibody (1:400 (v/v); CST, Shanghai, China), and mouse anti-MUC5AC antibody $\left(1: 150(\mathrm{v} / \mathrm{v})\right.$; GeneTex) overnight at $4{ }^{\circ} \mathrm{C}$. The sections were then incubated with goat antimouse horseradish peroxidase (HRP)-conjugated secondary antibody for $1 \mathrm{~h}$ at room temperature. 3,3'-Diaminobenzidine (DAB) was then used for color development, and dark brown staining was considered positive. The magnitude of staining was determined using the staining intensity and percentage of positive cells. 


\subsection{Scanning electron microscopy (SEM)}

The glutaraldehyde-fixed conjunctival and lacrimal duct samples were washed with PBS, then dehydrated in increasing ethanol concentrations $(50 \%$, $70 \%, 85 \%, 95 \%$, and $100 \% ; 15 \mathrm{~min} / \mathrm{step})$. The specimens were then postfixed for $2 \mathrm{~h}$ in $2 \%(0.02 \mathrm{~g} / \mathrm{ml})$ aqueous osmium tetroxide. After postfixation, the specimens were treated with a graded ethanol bath $(50 \%, 70 \%, 85 \%$, and $95 \% ; 15 \mathrm{~min} / \mathrm{step})$, and then incubated for $10 \mathrm{~min}$ in $100 \%$ hexamethyldisilazane for desiccation, and allowed to air-dry overnight. They were then attached to carbon stubs and coated with a $30 \mathrm{~nm}$-thick layer of platinum before being photographed with an SU8010 high resolution field emission SEM (Hitachi, Tokyo, Japan).

\subsection{Transmission electron microscopy (TEM)}

For TEM, the fresh conjunctival and lacrimal duct samples were carefully cut into $1 \mathrm{~mm}^{3}$ sections and immersed in $2.5 \%$ glutaraldehyde overnight. The glutaraldehyde-fixed tissues were then washed three times with PBS, dehydrated in increasing ethanol concentrations, and fixed for $2 \mathrm{~h}$ in $2 \%(0.02 \mathrm{~g} / \mathrm{ml})$ aqueous osmium tetroxide. The samples were dehydrated by being passed through a graded ethanol series, transferred to propylene oxide, and embedded in epoxy resin. Ultrathin sections (50-70 nm) were cut and stained with uranyl acetate and counterstained with lead citrate before examination under a JEM1200EX TEM (JEOL, Tokyo, Japan).

\subsection{Isolation and culture of epithelial cells from the conjunctival and lacrimal ducts}

The epithelial cells were isolated and cultured according to a slightly modified, previously reported method (Wei et al., 1993). Immediately after excision of the tissue, the fresh epithelial samples (the middle palpebral and fornix conjunctiva, bulbar conjunctiva, and lacrimal duct) were washed three times with Hank's balanced salt solution, containing $3 \times$ penicillinstreptomycin $(300 \mathrm{mg} / \mathrm{ml})$, and subjected to dispase II digestion (2400 U/L; Sigma-Aldrich, St. Louis, MO, USA), then treated at $37{ }^{\circ} \mathrm{C}$ for $1.5 \mathrm{~h}$ under $5 \% \mathrm{CO}_{2}$ and $95 \%$ air. Using a biological dissection microscope, the detached epithelium of the three parts was isolated by light scraping, and dissociated into single cells using a pipet. The isolated cells were then plated onto culture plates with Dulbecco's modified Eagle's/ Ham's 12 medium (DMEM/F12), supplemented with $10 \%(0.1 \mathrm{~g} / \mathrm{ml})$ fetal bovine serum, $100 \mathrm{U} / \mathrm{ml}$ penicillin, and $100 \mathrm{mg} / \mathrm{ml}$ streptomycin (all from GIBCO, USA), $2 \mathrm{mmol} / \mathrm{L}$ L-glutamine (Invitrogen, Carlsbad, CA, USA), and $20 \mathrm{ng} / \mathrm{ml}$ epidermal growth factor (EGF; Sigma-Aldrich) at $37{ }^{\circ} \mathrm{C}$ in a humidified atmosphere of $5 \% \mathrm{CO}_{2}$ and $95 \%$ air.

\subsection{Immunocytochemistry for epithelial phenotypes}

Primary rabbit palpebral and fornical conjunctival epithelial cells (PFCECs), bulbar conjunctival epithelial cells (BCECs), and lacrimal duct epithelial cells (LDECs) were seeded onto 24-well plates at a concentration of $8 \times 10^{4}$ cells $/ \mathrm{ml}$ and cultured for $24 \mathrm{~h}$. The cells were then fixed in $4 \%$ paraformaldehyde and incubated for $30 \mathrm{~min}$ at room temperature in blocking buffer that contained $1 \%(0.01 \mathrm{~g} / \mathrm{ml})$ bovine serum albumin and $0.2 \%$ Triton-X in PBS. The cells were then incubated with diluted primary antibodies (mouse anti-cytokeratin AE1+AE3 antibody, 1:50 (v/v); mouse anti-CK4 antibody, 1:50 (v/v); and mouse anti-MUC5AC antibody, 1:100 (v/v)) overnight at $4{ }^{\circ} \mathrm{C}$. The cells were washed three times in PBS, then treated for $1 \mathrm{~h}$ with the 1:200 (v/v) diluted secondary antibody (Alexa Fluor ${ }^{\circledR}$ 594-conjugated AffiniPure goat anti-mouse IgG; Jackson ImmunoResearch Laboratories, West Grove, PA, USA). The nuclei were counterstained with diamidino-2-phenylindole (DAPI) solution for $5 \mathrm{~min}$. Subsequently, the cells were washed with PBS and fluorescent images of the cells were obtained using an Olympus FV500 fluorescent microscope (Olympus, Tokyo, Japan).

\subsection{Proliferation analysis of cultured epithelial cells}

\subsubsection{Cell viability assay}

Cell proliferation ability was determined using a Cell Counting Kit-8 (CCK-8; Dojindo Laboratories, Kumamoto, Japan). Briefly, primary epithelial cells were plated onto 96-well plates (3000 cells/well). After 24,48 , and $72 \mathrm{~h}, 10 \mu \mathrm{l}$ of the CCK-8 solution and $90 \mu \mathrm{l}$ of culture medium were added to each well, and incubated at $37^{\circ} \mathrm{C}$ for $1-2 \mathrm{~h}$. The absorbance was measured at $450 \mathrm{~nm}$ using a 96-well iMark $^{\mathrm{TM}}$ microplate reader (Bio-Rad, Hercules, CA, USA). Six wells were used as replicates in every experiment, and the same treatment was performed in triplicate. 
2.7.2 Western blot analysis of proliferating cell nuclear antigen (PCNA) in cultured epithelial cells

The primary epithelial cells from three different areas were seeded onto 6 -well plates $\left(3 \times 10^{5}\right.$ cells/well $)$ and cultured for $72 \mathrm{~h}$. Then, total cellular protein was extracted using a Cell Total Protein Extraction Kit (Sangon, Shanghai, China). Western blot analysis was performed as previously described (Xie et al., 2014). The transferred polyvinylidene difluoride (PVDF) membranes were incubated with rabbit anti-PCNA antibody $(1: 2000, \mathrm{v} / \mathrm{v})$ and rabbit anti-glyceraldehyde3-phosphate dehydrogenase (GAPDH) antibody (1:2000, $\mathrm{v} / \mathrm{v}$; CST). After treating with peroxidase-conjugated goat anti-rabbit IgG (Jackson ImmunoResearch Laboratories; 1:5000, v/v), the cell extracts were visualized using enhanced chemiluminescence (ECL) reagents and exposed to X-ray film.

\subsection{Statistical analysis}

The results from three independent experiments were expressed as mean \pm standard deviation (SD).
Data were analyzed by one-way analysis of variance (ANOVA) with Bonferroni correction applied, using the SPSS for Windows software package (Ver. 16.0; SPSS Inc., Chicago, IL, USA). A value of $P<0.05$ was considered statistically significant.

\section{Results}

\subsection{Histological analysis of the $\mathrm{CjE}$ and the LDE}

Paraffin-embedded 5- $\mu \mathrm{m}$ sections from epithelial tissues were analyzed by $\mathrm{H} \& \mathrm{E}$ staining and immunohistochemistry (Fig. 1).

Stratified epithelium structures of 6-7 layers were observed in the eyelid margin, which changed to a double-layered epithelial structure in the palpebral, fornical, and bulbar conjunctiva. Several cell clusters in the posterior palpebral conjunctiva with nuclear/ cytoplasmic ratios were found. The LDE showed a similar stratified structure to that of the $\mathrm{CjE}$ (Fig. 1a). Pan-anti-cytokeratin AE1+AE3 is a broadly reactive

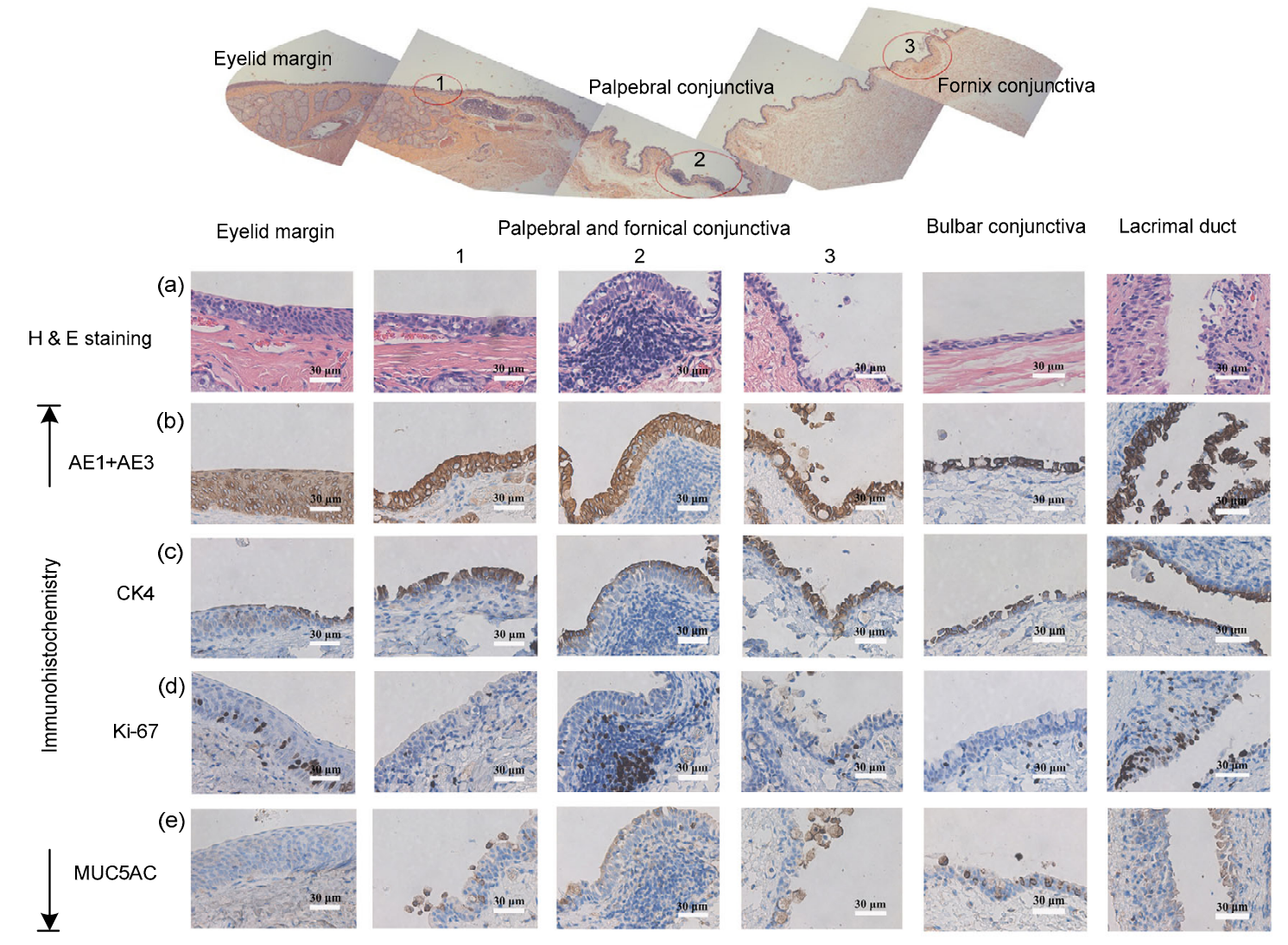

Fig. 1 Histologic observation of epithelium at eyelid margin, conjunctiva, and lacrimal duct with light microscopy Hematoxylin and eosin (H \& E)-staining (a); Immunohistochemical staining of cytokeratin AE1+AE3 (b), cytokeratin 4 (CK4) (c), $\mathrm{Ki}-67$ (d), and MUC5AC (e) 
marker, which recognizes epitopes present in most human epithelial tissues (Cooper et al., 1984). Superficial and basal cells of the eyelid margin, $\mathrm{CjE}$, and LDE all expressed cytokeratin AE1+AE3 (Fig. 1b). CK4, a non-cornifying squamous epithelial marker (Kasper, 1991), was strongly expressed in the cytoplasm of the superficial layers of the $\mathrm{CjE}$ and LDE, but no staining was detected in the eyelid margin, which might be cornified tissue (Fig. 1c). Ki-67, a classical marker for cell proliferation (Cattoretti et al., 1993), was rarely found in the epithelium of the $\mathrm{CjE}$, but strongly expressed by the basal layer of the eyelid margin, the bottom of the large cell cluster in the posterior palpebral conjunctiva (part 2), and the LDE (Fig. 1d). MUC5AC, a high molecular weight glycoprotein marker (Shatos et al., 2003), was expressed by the $\mathrm{CjE}$ and $\mathrm{LDE}$, and was absent in the eyelid margin epithelium (Fig. 1e). However, typical secretory MUC5AC-staining granules in and outside the differentiated goblet cells were observed in the anterior palpebral (part 1), fornical (part 3), and the bulbar $\mathrm{CjE}$. The epithelial cells in the posterior palpebra (part 2) and lacrimal duct diffusely expressed MUC5AC protein, with a relative decrease of differentiated goblet cells. The distribution of differentiated goblet cells with typical secretory granules was reduced in these proliferative areas, where Ki-67 was strongly expressed.

\subsection{SEM and TEM}

SEM was used to examine the different zones of the surfaces of the glutaraldehyde-fixed conjunctiva and the glutaraldehyde-fixed lacrimal duct samples (Fig. 2a). A typical stratified corneal layer was observed in the eyelid margin. The epithelial (nongoblet) cells comprised the palpebral and fornical conjunctival surface. They were polygonal, with a consistent size, but with slightly different shapes with obvious cell-cell borders. Among the non-goblet cells were numerous crater-like features, some of which were presumed to be goblet cell orifices. These orifices were located between the apices of the epithelial cells, and sometimes had strands of amorphous material discharged from them, which were presumed to be mucus. The BCECs had flat shapes attached to the surface, and the LDECs were densely arranged with columnar shapes. Under higher SEM magnification, all the epithelial cell surfaces were decorated with microvilli, and multiple forms of mucus were also present at the surfaces of the CjE. TEM showed cellular structures in different sections of the samples (Fig. 2b). Typical goblet cells discharging mucin granules were found in the anterior palpebral and fornical conjunctiva. Epithelial cells in the eyelid margin, palpebral, fornical and bulbar conjunctiva, and lacrimal duct were confirmed by TEM.

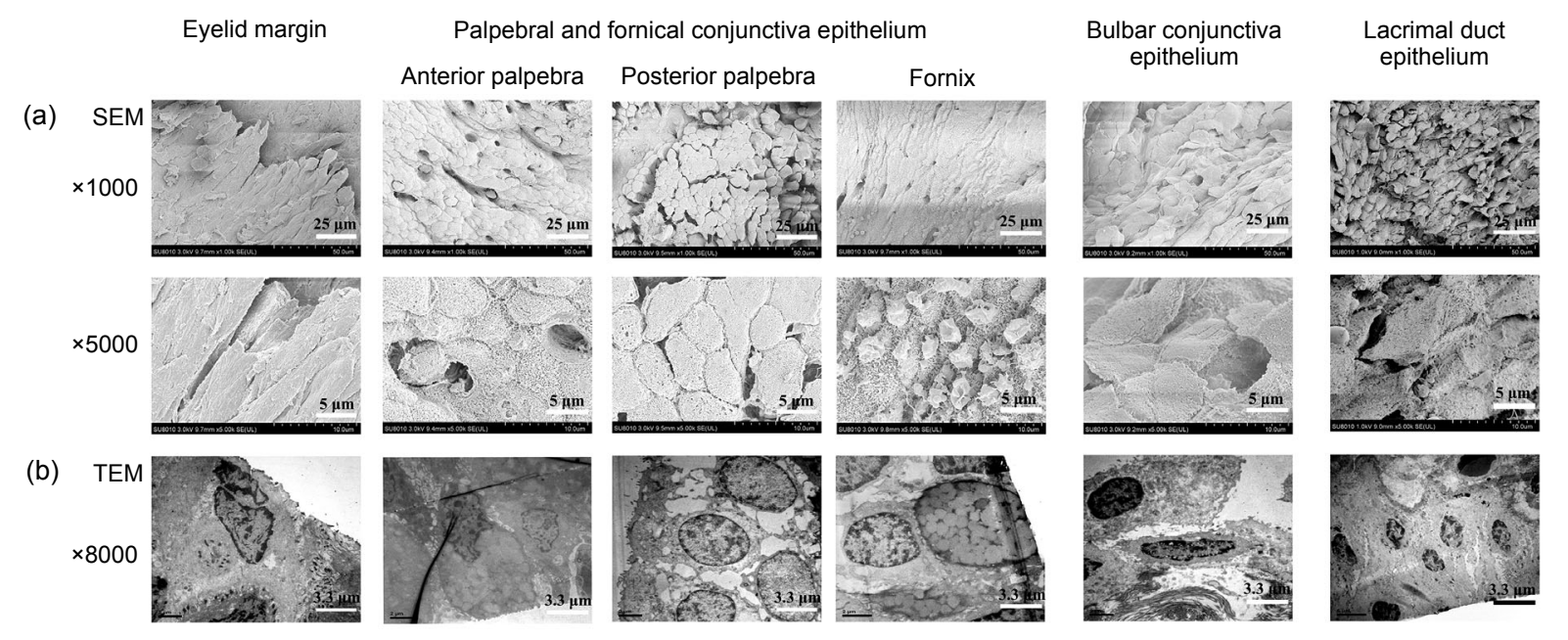

Fig. 2 Observation of epithelium at eyelid margin, conjunctiva, and lacrimal duct

(a) Scanning electron microscopy (SEM), low magnification $\times 1000$ and high magnification $\times 5000$; (b) Transmission electron microscopy $(\mathrm{TEM})$, magnification $\times 8000$ 


\subsection{Light microscopy and fluorescence immuno- cytochemistry}

Cells isolated from different epithelia were cultured in vitro using established methods (Wei et al., 1993). After 48-72 h, cellular morphology was observed by light microscopy (Fig. 3a).

The goblet cells in the $\mathrm{CjE}$ displayed a cobblestone morphology and highlighted background, and the BCECs had more stretched forms. Immunofluorescence of cytokeratin AE1+AE3, CK4, and MUC5AC was then used to characterize the cultured cells (Figs. 3b-3d). They expressed epithelium-specific cytokeratin $\mathrm{AE} 1+\mathrm{AE} 3$ and $\mathrm{CK} 4$, which was consistent with the histological results. Mucin-specific MUC5AC was also found in $\mathrm{CjE}$ cells and LDECs, although it was present only in specific cells of BCECs, which were presumed to be differentiated goblet cells.

\subsection{In vitro proliferation analysis of epithelial cells}

The proliferation profile of isolated epithelial cells used the methodology previously described. The same number of epithelial cells was plated onto 96well plates and cultured for 24,48 , and $72 \mathrm{~h}$. The viability of cells was measured by a CCK-8 kit. The epithelial cells from the palpebral and fornical conjunctiva and the lacrimal duct showed a significantly higher proliferative rate than BCECs $(P<0.05$; Fig. 4a).

Proliferation ability was further assessed by evaluating the PCNA in different epithelial cells. As described, the primary epithelial cells from three different areas were seeded onto 6-well plates $\left(3 \times 10^{5}\right.$ cells/well $)$ and cultured for $72 \mathrm{~h}$. Then, total cellular protein was extracted and PCNA was quantified by Western blot analysis (Fig. 4b). The relative

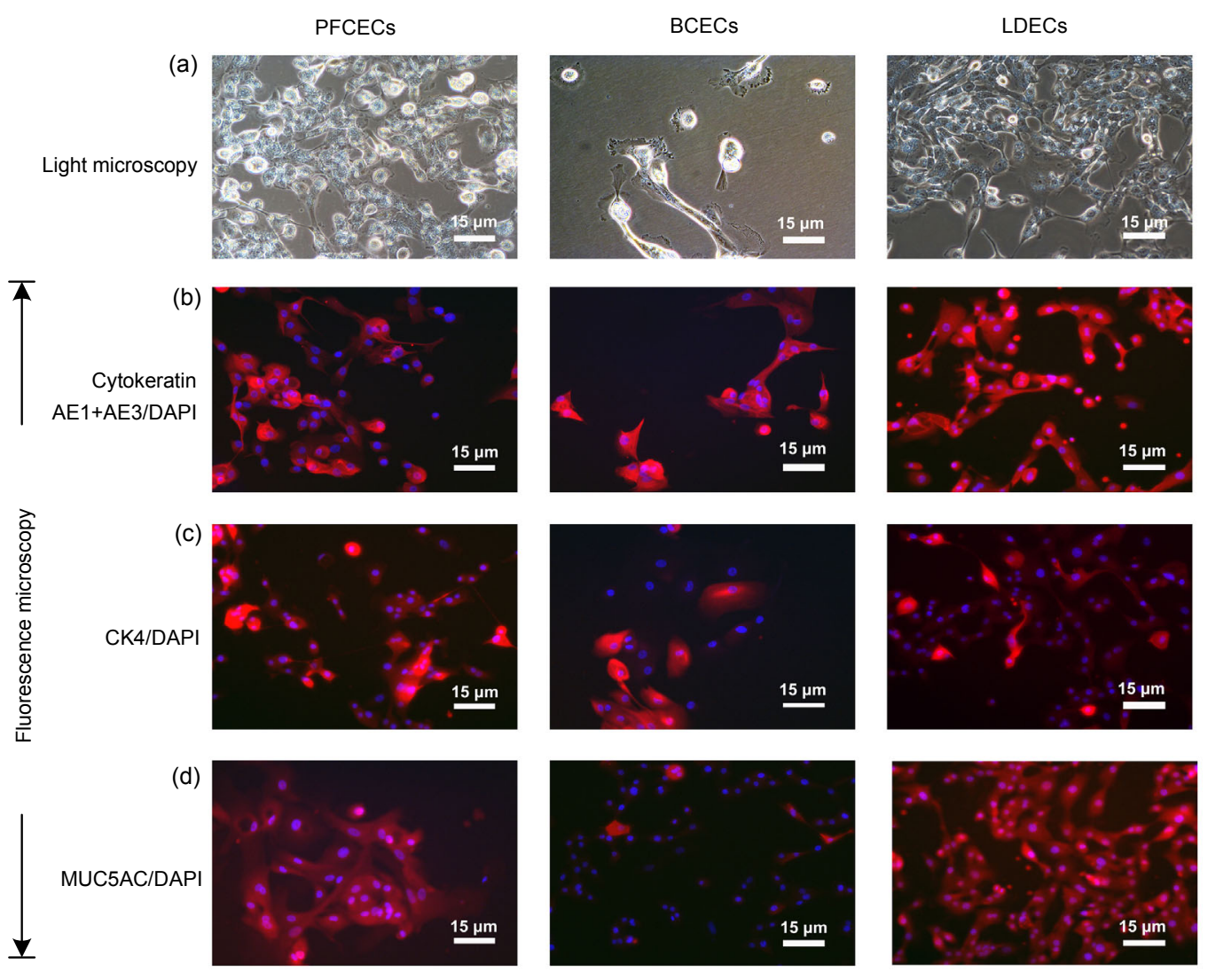

Fig. 3 Light microscopy and immunocytochemical evaluation of isolated palpebral and fornical conjunctival epithelial cells (PFCECs), bulbar conjunctival epithelial cells (BCECs), and lacrimal duct epithelial cells (LDECs) in vitro

Light morphology (a); Immunostained red for cytokeratin AE1+AE3 (b), cytokeratin 4 (CK4) (c), and MUC5AC (d). DAPI: diamidino-2-phenylindole (Note: for interpretation of the references to color in this figure legend, the reader is referred to the web version of this article) 
(a)

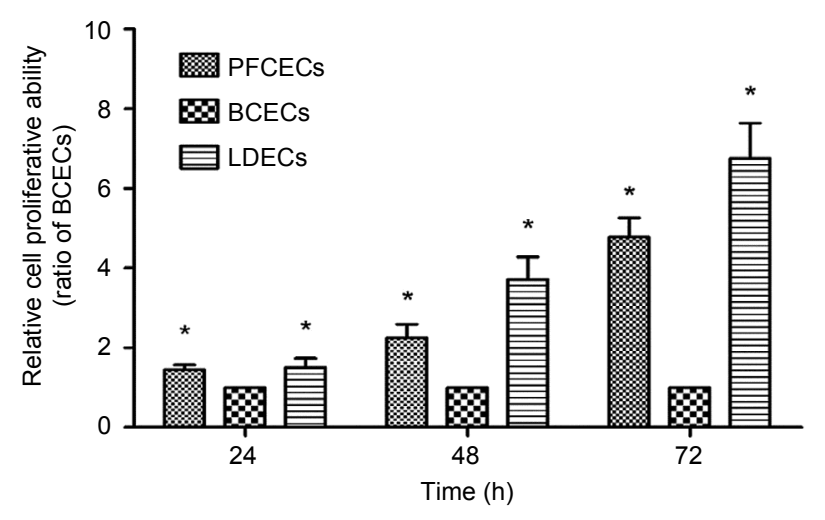

(b)

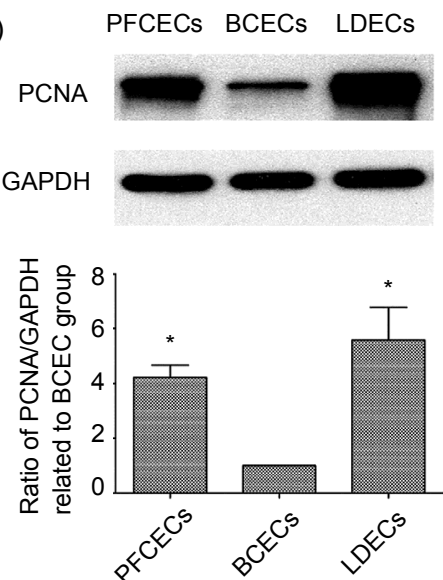

Fig. 4 Proliferative ability and proliferating cell nuclear antigen (PCNA) expression of cultured palpebral and fornical conjunctival epithelial cells (PFCECs), bulbar conjunctival epithelial cells (BCECs), and lacrimal duct epithelial cells (LDECs)

(a) Proliferative ability of epithelial cells after cultured in vitro for 24,48 , and $72 \mathrm{~h}$, respectively. Results, expressed as ratios of BCECs, are given in terms of the mean \pm standard deviation (SD) ( $n=6$ for three individual cultures). (b) Western blotting analysis of PCNA contents in epithelial cells after cultured in vitro for $72 \mathrm{~h}$. Increased expression levels of PCNA, normalized with glyceraldehyde-3-phosphate dehydrogenase (GAPDH), were observed in the PFCECs and LDECs, respectively. Results of protein relative ratio are presented as mean $\pm \mathrm{SD}$ ( $n=3$ for three individual experiments). The significance of differences $\left({ }^{*} P<0.05\right)$ was determined with respect to the BCEC group by Student's $t$-test

PCNA levels of PFCECs and LDECs were 4.21 0.45 times and 5.58 \pm 1.20 times the value of BCECs, respectively.

\section{Discussion}

Recently, the use of tissue-engineered epithelial transplantation has revolutionized the treatment of patients with severe ocular surface disease (Ang et al., 2010; Ricardo et al., 2013). The novel use of cultivated autologous conjunctival epithelial transplantation for reconstructing the corneal surface and treating limbal stem cell deficiency is a promising treatment. It has the advantages of safety, a decreased risk of rejection and infection, and no need for long-term treatment with steroids for immunosuppression (Tanioka et al., 2006; Ang et al., 2010). Mucosal integrity of the lacrimal duct system is the key to success using traditional methods of surgery for the correction of lacrimal pathway dysfunctions (traumatic damage, stenosis, and obstruction) (Takahashi et al., 2013; Baran et al., 2014). The application of tissue-engineered technology is promising for recovering the epithelial structure in severe lacrimal duct disease with extensively damaged LDE. However, before tissue-engineered LDE construction can be successful, autologous seed cells with similar biological characteristics to LDECs are needed. The ideal seed cells for tissue engineering should possess the necessary biological functions, and should be easily obtained from in vivo sources and massively proliferated in vitro. The objective of the present study was to identify suitable seed cells for constructing tissue-engineered LDECs.

The LDE is continuous with the $\mathrm{CjE}$, which covers the ocular surface, establishing a stable tear film for clear vision and protecting the eye from external insults. We characterized the distribution of epithelial cells in tissue using cytokeratin AE1+AE3, a broadly reactive epithelial marker, and $\mathrm{CK} 4$, a non-cornifying squamous epithelial marker. Cytokeratin AE1+AE3 was expressed by all epithelial cells in the eyelid margin, conjunctiva, and lacrimal duct. There is strong CK4 expression in superficial conjunctival cells, whereas the basal cells show no immunoreactivity for this marker (Risse Marsh et al., 2002). Similar results were obtained in the present study. Moreover, using CK4 immunostaining, the superficial epithelial cells in the lacrimal duct were first shown to be similar to non-cornifying squamous epithelial cells.

Mucins are considered to be heavily glycosylated glycoproteins, which are secreted from goblet 
cells within the conjunctiva (Inatomi et al., 1996; Wei et al., 1996; Gipson, 2004). Lacrimal ducts also express a broad spectrum of mucins, which enhance tear transport and antimicrobial defense (Paulsen et al., 2003). The present study showed that MUC5AC, a glycoprotein marker, was coexpressed by epithelium of the conjunctiva and lacrimal duct, but in a different pattern. Secretory particles containing mucins were found inside and outside the differentiated goblet cells of the conjunctiva, but not in the LDECs.

Electron microscopy provides objective evaluation and detailed information on the conjunctiva and lacrimal duct. As visualized by SEM, epithelial cells from the conjunctiva and lacrimal duct are normally decorated with very conspicuous microvilli. Images of the epithelial surface by SEM showed cells with a range of small to large openings or orifices. Numerous orifices, located between the apices of the epithelial cells, were distributed in the anterior palpebral, fornical, and bulbar conjunctivae, but fewer orifices in the posterior palpebral regions, and never in the lacrimal duct. Strands of amorphous material secreted from these orifices were presumed to be mucus, which was similar to findings obtained on other mucous membrane surfaces (Siew and Goldstein, 1981; Skrzypek et al., 2005). SEM revealed intracellular structures of palpebral and fornical conjunctival goblet cells discharging mucus, which consisted of small secretory particles. These results further confirmed histological evaluations of the conjunctiva, which showed that in both rabbits and humans, these differentiated goblet cells are single entities that discharge mucous materials to maintain wetness of the ocular surface (Gipson and Tisdale, 1997; Doughty, 2013). However, typical goblet cells were not found in the LDE of rabbits.

It has been hypothesized that, for the purpose of self-renewal, stem cells reside in the conjunctiva and generate transient amplifying cells, and also differentiate into specialized cell types, including goblet cells and non-goblet cells, to maintain the survival and health of the conjunctiva (Wolosin et al., 2004). However, the localization of stem cells is still controversial. In the present study, Ki-67, a marker for cell proliferation, was detected at the base of specific dome structures, under the epithelium in the posterior palpebral conjunctiva, which contained cells with high nuclear/cytoplasmic ratios. Epithelial markers, cytokeratin AE1+AE3 and CK4, were poorly expressed in these cells. In addition, differentiated goblet cells with secretory mucins were also found, but in lesser amounts, in the posterior palpebral $\mathrm{CjE}$ with dome structures, when compared with other areas of the conjunctiva. Although there is limited direct evidence in the present study that the specific dome structures contained poorly differentiated cells that could be considered "stem cell territory," these findings might nonetheless assist in the selection of candidate cells for tissue engineering.

In addition, we successfully isolated and cultured cells from the palpebral and fornical $\mathrm{CjE}$, bulbar $\mathrm{CjE}$, and LDE. Proliferation analyses indicated that PFCECs and LDECs had a significantly higher in vitro rate of proliferation than BCECs. We confirmed our results with another important characteristic of stem cells, which was their potential to remain highly proliferative. These results are also consistent with another study (Su et al., 2011), reporting that rabbit palpebral epithelial cells grow faster and have more proliferative cells (likely originating from cancer stem cells with higher in vitro colony forming rates than the other parts of the conjunctiva).

In conclusion, PFCECs are anatomically connected to LDECs. They have similar morphological characteristics, immune phenotypes, and proliferation features. PFCECs are therefore potential candidate cells to replace LDECs in tissue engineering procedures to treat lacrimal duct diseases.

\section{Compliance with ethics guidelines}

Chen XIE, Xiu-yi LI, and Hong-guang CUI declare that they have no conflict of interest.

All institutional and national guidelines for the care and use of laboratory animals were followed.

\section{References}

Ang, L.P., Tanioka, H., Kawasaki, S., et al., 2010. Cultivated human conjunctival epithelial transplantation for total limbal stem cell deficiency. Invest. Ophthalmol. Vis. Sci., 51(2):758-764. [doi:10.1167/iovs.09-3379]

Avgitidou, G., Koch, K.R., Cursiefen, C., et al., 2015. Current aspects of eyelid, lacrimal and orbital surgery in childhood. Der Ophthalmologe, 112(2):102-109 (in German). [doi:10.1007/s00347-014-3054-8]

Baran, F., Kelly, J.P., Finn, L.S., et al., 2014. Evaluation and treatment of failed nasolacrimal duct probing in Down syndrome. J. AAPOS, 18(3):226-231. [doi:10.1016/j. jaapos.2013.12.018] 
Bonilha, V.L., Rayborn, M.E., Shadrach, K., et al., 2006. Characterization of semenogelin proteins in the human retina. Exp. Eye Res., 83(1):120-127. [doi:10.1016/j.exer. 2005.11.011]

Cattoretti, G., Orazi, A., Gerdes, J., 1993. Proliferating normal bone marrow cells do stain for Ki-67 antigen. $B r . J$. Haematol., 85(4):835-836. [doi:10.1111/j.1365-2141. 1993.tb03238.x]

Cooper, D., Schermer, A., Pruss, R., et al., 1984. The use of aIF, AE1, and AE3 monoclonal antibodies for the identification and classification of mammalian epithelial keratins. Differentiation, 28(1):30-35. [doi:10.1111/j.1432-0436. 1984.tb00263.x]

Doughty, M.J., 2013. Assessment of goblet cell orifice distribution across the rabbit bulbar conjunctiva based on numerical density and nearest neighbors analysis. Curr. Eye Res., 38(2):237-251. [doi:10.3109/02713683.2012.754901]

Gipson, I.K., 2004. Distribution of mucins at the ocular surface. Exp. Eye Res., 78(3):379-388. [doi:10.1016/S0014-4835 (03)00204-5]

Gipson, I.K., Tisdale, A.S., 1997. Visualization of conjunctival goblet cell actin cytoskeleton and mucin content in tissue whole mounts. Exp. Eye Res., 65(3):407-415. [doi:10. 1006/exer.1997.0351]

Goldstein, S.M., Katowitz, J.A., Syed, N.A., 2006. The histopathologic effects of balloon dacryoplasty on the rabbit nasolacrimal duct. J. AAPOS, 10(4):333-335. [doi:10. 1016/j.jaapos.2006.03.004]

Inatomi, T., Spurr-Michaud, S., Tisdale, A.S., et al., 1996. Expression of secretory mucin genes by human conjunctival epithelia. Invest. Ophthalmol. Vis. Sci., 37(8): 1684-1692.

Jäger, K., Wu, G., Sel, S., et al., 2007. MUC16 in the lacrimal apparatus. Histochem. Cell Biol., 127(4):433-438. [doi:10. 1007/s00418-006-0246-6]

Kam, J.K., Cheng, N.M., Sarossy, M., et al., 2014. Nasolacrimal duct screening to minimize post-cataract surgery endophthalmitis. Clin. Exp. Ophthalmol., 42(5): 447-451. [doi:10.1111/ceo.12244]

Kasper, M., 1991. Heterogeneity in the immunolocalization of cytokeratin specific monoclonal antibodies in the rat eye: evaluation of unusual epithelial tissue entities. Histochemistry, 95(6):613-620. [doi:10.1007/BF00266749]

Langer, R., Vacanti, J.P., 1993. Tissue engineering. Science, 260(5110):920-926. [doi:10.1126/science.8493529]

Lopez, P.F., Beldavs, R.A., al-Ghamdi, S., et al., 1993. Pneumococcal endophthalmitis associated with nasolacrimal obstruction. Am. J. Ophthalmol., 116(1):56-62. [doi:10.1016/S0002-9394(14)71744-1]

Mukherjee, B., Dhobekar, M., 2013. Traumatic nasolacrimal duct obstruction: clinical profile, management, and outcome. Eur. J. Ophthalmol., 23(5):615-622. [doi:10.5301/ ejo.5000256]

Nakamura, T., Kinoshita, S., 2003. Ocular surface reconstruction using cultivated mucosal epithelial stem cells. Cornea, 22(7):S75-S80. [doi:10.1097/00003226-200310
001-00011]

Nakamura, T., Endo, K., Cooper, L.J., et al., 2003. The successful culture and autologous transplantation of rabbit oral mucosal epithelial cells on amniotic membrane. Invest. Ophthalmol. Vis. Sci., 44(1):106-116. [doi:10. 1167/iovs.02-0195]

Paulsen, F.P., Corfield, A.P., Hinz, M., et al., 2003. Characterization of mucins in human lacrimal sac and nasolacrimal duct. Invest. Ophthalmol. Vis. Sci., 44(5): 1807-1813. [doi:10.1167/iovs.02-0744]

Pellegrini, G., Traverso, C.E., Franzi, A.T., et al., 1997. Longterm restoration of damaged corneal surfaces with autologous cultivated corneal epithelium. Lancet, 349(9057): 990-993. [doi:10.1016/S0140-6736(96)11188-0]

Prabhasawat, P., Tseng, S.C., 1998. Frequent association of delayed tear clearance in ocular irritation. Br. J. Ophthalmol., 82(6):666-675. [doi:10.1136/bjo.82.6.666]

Ricardo, J.R., Cristovam, P.C., Filho, P.A., et al., 2013. Transplantation of conjunctival epithelial cells cultivated ex vivo in patients with total limbal stem cell deficiency. Cornea, 32(3):221-228. [doi:10.1097/ICO.0b013e3182 5034be]

Risse Marsh, B.C., Massaro-Giordano, M., Marshall, C.M., et al., 2002. Initiation and characterization of keratinocyte cultures from biopsies of normal human conjunctiva. Exp. Eye Res., 74(1):61-69. [doi:10.1006/exer.2001.1099]

Shatos, M.A., Rios, J.D., Horikawa, Y., et al., 2003. Isolation and characterization of cultured human conjunctival goblet cells. Invest. Ophthalmol. Vis. Sci., 44(6):24772486. [doi:10.1167/iovs.02-0550]

Siew, S., Goldstein, M.L., 1981. Scanning electron microscopy of mucosal biopsies of the human upper gastrointestinal tract. Scan. Electron. Microsc., 4:173-181.

Skrzypek, T., Valverde Piedra, J.L., Skrzypek, H., et al., 2005. Light and scanning electron microscopy evaluation of the postnatal small intestinal mucosa development in pigs. $J$. Physiol. Pharmacol., 56(Suppl. 3):71-87.

Su, L., Cui, H., Xu, C., et al., 2011. Putative rabbit conjunctival epithelial stem/progenitor cells preferentially reside in palpebral conjunctiva. Curr. Eye Res., 36(9):797-803. [doi:10.3109/02713683.2011.593727]

Takahashi, Y., Nakamura, Y., Kakizaki, H., 2013. Dacryoendoscopic findings in the lacrimal passage in failed dacryocystorhinostomy. Ophthal. Plast. Reconstr. Surg., 29(5):373-375. [doi:10.1097/IOP.0b013e31829a72f9]

Takahashi, Y., Nakamura, Y., Kakizaki, H., 2015. Eight-flap anastomosis in external dacryocystorhinostomy. $\mathrm{Br} . \mathrm{J}$. Ophthalmol., 99(11):1527-1530. [doi:10.1136/bjophthalmol2014-306570]

Tanioka, H., Kawasaki, S., Yamasaki, K., et al., 2006. Establishment of a cultivated human conjunctival epithelium as an alternative tissue source for autologous corneal epithelial transplantation. Invest. Ophthalmol. Vis. Sci., 47(9): 3820-3827. [doi:10.1167/iovs.06-0293]

Wei, Z.G., Wu, R.L., Lavker, R.M., et al., 1993. In vitro growth and differentiation of rabbit bulbar, fornix, and 
palpebral conjunctival epithelia. Implications on conjunctival epithelial transdifferentiation and stem cells. Invest. Ophthalmol. Vis. Sci., 34(5):1814-1828.

Wei, Z.G., Sun, T.T., Lavker, R.M., 1996. Rabbit conjunctival and corneal epithelial cells belong to two separate lineages. Invest. Ophthalmol. Vis. Sci., 37(4):523-533.

Wolosin, J.M., Budak, M.T., Akinci, M.A., 2004. Ocular surface epithelial and stem cell development. Int. J. Dev. Biol., 48(8-9):981-991. [doi:10.1387/ijdb.041876jw]

Xie, C., Li, X., Tong, J., et al., 2014. Effects of white light-emitting diode (LED) light exposure with different correlated color temperatures (CCTs) on human lens epithelial cells in culture. Photochem. Photobiol., 90(4): 853-859. [doi:10.1111/php.12250]

\section{中文概要}

题 目: 潜在候选细胞构建组织工程的泪道上皮: 兔的组 织学和细胞学研究

目 的: 泪道上皮细胞的损伤和缺陷会导致各种各样的泪 道疾病。本研究的目的是探寻潜在的候选细胞构 建组织工程泪道上皮。
创新点: 首次在活体及离体水平上比较不同部位的结膜上 皮细胞与泪道上皮细胞的生物组织特性的相似 性, 以获得构建组织工程泪道上皮的理想候选细 胞。

方 法: 取雄性新英格兰大白兔的不同区域的结膜及泪道 组织, 用于组织生物学评价。采用苏木精-伊红染 色法及免疫组织化学鉴定细胞角蛋白 AE1+AE3、 细胞角蛋白 4、人细胞增殖细胞核核抗原 Ki-67 及黏蛋白 MUC5AC（图 1)。场发射扫描电镜及 投射电镜观察不同的上皮表层形态及细胞结构

(图 2)。此外, 用酶化法将上皮细胞从组织分 离, 并用特定的抗体鉴定细胞（图 3)。比较不 同区域来源的结膜上皮细胞及泪道上皮细胞的 离体增殖能力和细胞表达的增殖细胞核核抗原 （PCNA）的差异（图 4）。

结 论: 新英格兰大白兔的睑结膜上皮细胞在组织结构上 与泪道上皮细胞更加接近。它们拥有相似的形态 特征、蛋白免疫表型及增殖活性。睑结膜部的结 膜上皮细胞可能是潜在的候选细胞用于构建组 织工程的泪道上皮, 进而用于治疗泪道疾病。

关键词: 结膜上皮细胞; 泪道上皮细胞; 组织工程; 细胞 角蛋白; 黏蛋白; 人增殖细胞核核抗原 